\title{
A novel cost-effective parallel narrowband ANC system with local secondary-path estimation
}

\author{
Riccardo Delegà, Giancarlo Bernasconi ${ }^{\mathrm{a}}$, Luigi Piroddi ${ }^{\mathrm{a}, *}$ \\ ${ }^{a}$ Politecnico di Milano, Dipartimento di Elettronica, Informazione e Bioingegneria \\ Via Ponzio 34/5, 20133 Milano, Italy.
}

\begin{abstract}
Many noise reduction applications are targeted at multi-tonal disturbances. Active noise control (ANC) solutions for such problems are generally based on the combination of multiple adaptive notch filters. Both the performance and the computational cost are negatively affected by an increase in the number of controlled frequencies. In this work we study a different modeling approach for the secondary path, based on the estimation of various small local models in adjacent frequency subbands, that greatly reduces the impact of reference-filtering operations in the ANC algorithm. Furthermore, in combination with a frequency-specific step size tuning method it provides a balanced attenuation performance over the whole controlled frequency range (and particularly in the high end of the range). Finally, the use of small local models is greatly beneficial for the reactivity of the online secondary path modeling algorithm when the characteristics of the acoustic channels are time-varying. Several simulations are provided to illustrate the positive features of the proposed method compared to other well-known techniques.
\end{abstract}

Keywords: Active noise control; Adaptive digital filters; Adaptive algorithms; Narrowband noise; Adaptive notch filters; Model identification.

\section{Introduction}

Rotating components in industrial machinery emit high-level low-frequency periodic noise, that may cause severe physiological and psychological distress on human beings in case of long periods of exposure. Traditional passive noise-control techniques such as enclosures, barriers and silencers are

\footnotetext{
*Corresponding author, voice (39) 022399 3556, fax (39) 0223993412.

Email addresses: riccardo.delega@mail.polimi.it (Riccardo Delegà), giancarlo.bernasconi@polimi.it (Giancarlo Bernasconi), luigi.piroddi@polimi.it (Luigi Piroddi) 
not particularly suited for low-frequency noise control, because the noise wavelengths are large with respect to the thickness of the acoustic absorbers typically allowed in these applications. A viable alternative is provided by active noise control (ANC) techniques. ANC exploits the superposition principle, whereby the undesired noise is cancelled (or attenuated) by injecting an anti-noise signal of equal amplitude and opposite phase (destructive interference) using a secondary source, such as a loudspeaker $[1,2]$. The anti-noise signal is generated by an adaptive filter fed with a reference signal, that has to be well correlated with the noise, and tuned using algorithms of the Least Mean Squares (LMS) family. In particular, the basic algorithm is a variation of the LMS algorithm, denoted FxLMS, which suitably takes into account the secondary path (i.e., the measurement and control chain).

Since most of the spectral power in the applications mentioned above is concentrated in tonal components, specific ANC techniques have been developed to address periodic noise. Adaptive notch filters employ synthesized sine waves as reference signals, tuned to the noise frequencies (either known in advance or estimated), and suitably modulate them in amplitude and phase to track the disturbance $[1,3]$. The multi-tonal case is typically addressed by combining in parallel several single-tone adaptive notch filters, each fed by a sinusoid at a given frequency. The parameters of each adaptive notch filter are tuned with the FxLMS algorithm, which implies that each sinusoidal reference signal be convolved with an estimate of the secondary path in order to obtain the filtered-reference signals used in the algorithm [4]. Since the acoustic environment in practical applications is often time varying, narrowband ANC systems typically include a subsystem that updates the secondary path estimate during system operation to avoid performance degradation and instability issues.

The basic parallel-form narrowband ANC system described above suffers from four problems: (i) A single error signal containing the contribution of all tonal components is used to update each adaptive notch filter unit. (ii) The filtering operations on the reference signals have an increasing impact in terms of computational load as the number of controlled frequencies increases, since the FIR filters used to model the secondary path need a large number of coefficients to achieve sufficient modeling accuracy; (iii) The online modeling subsystem is slow to react to changes in the secondary path, mainly because of the large number of parameters of the employed models; (iv) The choice of a suitable step size for each adaptive notch filter unit is a crucial - and not trivial - 
task in practice.

The first issue is a known source of degradation of the performance of the multi-tonal parallel system with respect to the single-tone case, both in terms of convergence speed and noise reduction [5]. The main reason for this is the mutual interference between the different tonal components originated by the error signal. To tackle this issue a parallel narrowband ANC system was proposed in [5] that employed a bank of bandpass filters to separate the frequency components of the error and drive each adaptive notch filter with the appropriate error component. Though such filters were designed to be delayless (at least at the center of the pass-band), it is argued in [6] that they exhibit a peak in the group delay which increases as their bandwidth is decreased. Simulations showed that this overlooked issue limits the convergence speed to the extent that the conventional narrowband controller still converges faster. For this reason, this method is not further considered in the comparison analysis provided in this work.

Regarding issue (ii), several solutions have been proposed in the literature with the aim of reducing the computational burden of the parallel-form narrowband ANC system, while at the same time yielding the same performance in terms of noise canceling and speed of convergence. One first idea to account for the secondary path without incurring in large high computational costs dates back to 1989, when Ziegler developed a narrowband ANC system that compensates the phase shift introduced by the secondary path at the controlled frequencies with a suitable number of unit delay blocks, so that the reference-filtering operations only consist in delaying the reference signal by a given number of samples [7]. While computationally very efficient, this solution suffers from convergence speed and noise canceling performance issues, due to the estimation errors on the secondary path. More recently [8] Xiao proposed a system where the referencefiltering operations are reduced to one, by summing the reference signals first, convolving their sum with the secondary-path estimate, and finally retrieving the individual filtered reference signals by filtering the convolved signal through a bank of bandpass filters. Xiao's system only performs one reference-filtering operation regardless of the number of controlled frequencies and often achieves the same convergence-speed and noise canceling properties of the basic parallel-form narrowband ANC system. However, the correct design of the bandwidth of the bandpass filters is critical for an appropriate separation of the filtered reference signals, and becomes increasingly difficult as the controlled frequencies get closer. If the bandwidth-control parameter is too small, system 
instability may occur. Conversely, if it is too large, the system may exhibit slow convergence.

We here propose a novel cost-effective approach for the compensation of the secondary path in multi-tonal narrowband ANC systems. Rather than using a single global high-order FIR model, we investigate how multiple local lower-order FIR models (called local secondary paths) may be employed to obtain the filtered reference signals. A computational analysis establishes that the proposed system reduces the number of required computations with respect to the original system. Simulations show that the proposed system offers comparable convergence speed and noise canceling performance with respect to the basic multi-tonal parallel-form system and that it outperforms Ziegler's and Xiao's systems in situations that are critical for those systems. Furthermore, simulations show that the online modeling of local secondary paths is more reactive to changes in the secondary path than the global online modeling system thanks to the low order of the local estimates (issue (iii) above). This increased reactivity makes the system less prone to instability if the characteristics of the environment change rapidly. Finally, an empirical step size-correction law is proposed to automatically tune the step size of each adaptive filter in the parallel-form narrowband ANC system without resorting to normalized FxLMS or trial and error (issue (iv) above).

The rest of the paper is organized as follows. Section 2 reviews narrowband ANC systems. Section 3 presents the novel ANC scheme, which is evaluated in various simulation tests in Section 4 . Section 5 provides a brief computational analysis of the proposed method. Finally, some conclusions are provided in Section 6.

\section{Narrowband ANC systems}

\subsection{The basic parallel-form multi-tonal narrowband ANC system}

The basic narrowband ANC system based on FxLMS was introduced by Glover in [9] back in 1977 and is depicted in Figure 1. The disturbance is a multi-tonal signal, defined as follows:

$$
d(n)=\sum_{i=0}^{M-1}\left(a_{i} \cos \left(2 \pi f_{i} n\right)+b_{i} \sin \left(2 \pi f_{i} n\right)\right),
$$

where $M$ is the number of tonal components at frequencies $f_{i}, i=1, \ldots, M$, and $a_{i}$ and $b_{i}$, $i=1, \ldots, M$, are given coefficients. The control objective is to eliminate (or, more realistically,

reduce) the tonal components at the error measurement point, by injecting a secondary signal which interferes negatively with the multi-tonal disturbance. 


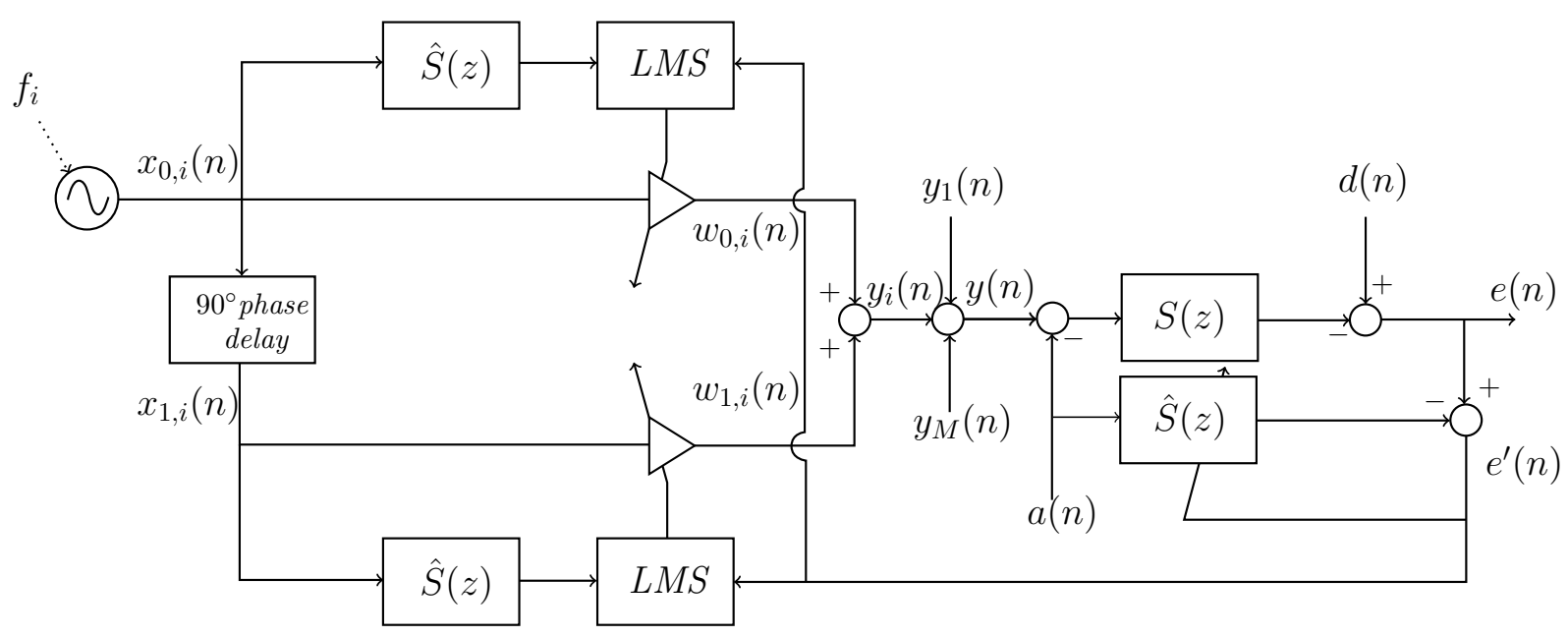

Figure 1: Basic narrowband ANC system with online secondary path modeling.

Consider first the plain control problem, disregarding momentarily the secondary path estimation loop (i.e., assume that signal $a(n)$ in Figure 1 is equal to zero). Assuming that the disturbance frequencies are known, the control consists in suitably modulating some reference signals tuned at the mentioned frequencies to produce the desired secondary signal. Essentially, a pair of sinusoidal signals in quadrature are used as reference signals for each frequency $\left(x_{0, i}(n)=A \cos \left(2 \pi f_{i} n\right)\right.$ and $\left.x_{1, i}(n)=A \sin \left(2 \pi f_{i} n\right)\right)$, and the corresponding coefficients $\left(w_{0, i}(n)\right.$ and $\left.w_{1, i}(n)\right)$ are tuned with the FxLMS algorithm to adjust the magnitude and phase of the produced tonal anti-noise signal $y_{i}(n)$. This signal is filtered by the secondary path $S(z)$ (i.e., the measurement and control chain), to eventually produce the signal $y_{i}^{\prime}(n)=y_{i}(n) * s(n)$ that actually compensates the $i$ th frequency component of the original noise disturbance $d(n)$ at the error microphone. In case of multiple tonal components, several signals $y_{i}, i=1, \ldots, M$, are constructed in the explained way and summed together:

$$
y(n)=\sum_{i=1}^{M} y_{i}(n)=\sum_{i=1}^{M}\left(w_{0, i}(n) x_{0, i}(n)+w_{1, i}(n) x_{1, i}(n)\right) .
$$

The controller uses the signal

$$
e^{\prime}(n)=e(n)=d(n)-y^{\prime}(n)=d(n)-y(n) * s(n)
$$

to tune the adaptive coefficients $w_{0, i}(n)$ and $w_{1, i}(n), i=1, \ldots, M$, according to the FxLMS update 
rule

$$
\begin{aligned}
& w_{0, i}(n+1)=w_{0, i}(n)+\mu_{i} x_{0, i}^{\prime}(n) e^{\prime}(n), \\
& w_{1, i}(n+1)=w_{1, i}(n)+\mu_{i} x_{1, i}^{\prime}(n) e^{\prime}(n),
\end{aligned}
$$

for $i=1, \ldots, M$, where $x_{0, i}^{\prime}(n)$ and $x_{1, i}^{\prime}(n)$ are the filtered reference signals through the secondarypath estimate $\hat{S}(z)$ and $\mu_{i}$ are the step sizes of each narrowband ANC block.

In general, it is not safe to assume that the secondary path $S(z)$ should remain constant during the operation of the ANC system, because of the time-varying characteristics of the acoustic channel. Accounting incorrectly for the secondary path can jeopardize the stability of the ANC algorithm in the presence of phase errors exceeding $90^{\circ}$ [1]. To avoid this from happening, an online-modeling subsystem keeps track of these changes and adapts the secondary-path model accordingly. For this purpose, a low-energy broadband excitation signal $a(n)$ (typically, a white Gaussian noise) is injected into the system at the control point (see Figure 1) and used as input to the secondary path model $\hat{S}(z)$ (typically represented as an FIR filter $\hat{\mathbf{s}}(n)=\left[\begin{array}{lll}\hat{s}_{0}(n) \ldots \hat{s}_{L^{\prime}-1}(n)\end{array}\right]^{T}$ ) as well. This modifies the error signal to $e(n)=d(n)-y^{\prime}(n)+a^{\prime}(n)=d(n)-y^{\prime}(n)+a(n) * s(n)$, $s(n)$ being the finite impulse response sequence of the true secondary path. The secondary path model is updated using the signal

$$
\begin{aligned}
e^{\prime}(n) & =e(n)-\hat{a}^{\prime}(n)=e(n)-a(n) * \hat{s}(n)= \\
& =d(n)-y^{\prime}(n)+a(n) *(s(n)-\hat{s}(n))
\end{aligned}
$$

with the LMS update law:

$$
\hat{\mathbf{s}}(n+1)=\hat{\mathbf{s}}(n)+\mu_{s} \mathbf{a}(n) e^{\prime}(n),
$$

where $\mathbf{a}(n)=\left[\begin{array}{lll}a(n) & \ldots a\left(n-L^{\prime}+1\right)\end{array}\right]^{T}$ is the excitation signal vector and $\mu_{s}$ is the online-modeling step size. Provided that the injected auxiliary signal $a(n)$ is uncorrelated with $d(n)-y^{\prime}(n)$ (and that the step size is sufficiently small), this LMS will converge to a solution minimizing the secondary path estimation error $s(n)-\hat{s}(n)$. Regarding the noise cancellation problem, notice that signal $a(n)$ does not affect the controller updating in the case of perfect secondary path modeling, since in that case $\left.e^{\prime}(n)=d(n)-y^{\prime}(n)\right)$, and in general it only slightly worsens the cancellation performance (provided that it is sufficiently small).

This system suffers from two problems that originate from the need to employ high-order FIR filters for accurate secondary-path estimation over a frequency band large enough to cover all 
tonal components (the order $L^{\prime}$ of the secondary-path model is normally quite large, with 128 taps or more [8]). First, the reference-filtering operations require $M L^{\prime}$ multiplications per iteration, a cost that grows linearly with the number of controlled frequencies and can easily become the computational bottleneck of an otherwise efficient controller. Second, since the step size of the online-modeling subsystem is inversely proportional to the order of the secondary-path model, the convergence speed of the same subsystem is negatively affected by a large value of $L^{\prime}$. This may in turn jeopardize the stability of the overall system, if $\hat{S}(z)$ is not updated fast enough to cover large phase mismatches. A further issue is related to the different convergence speed associated to the various adaptive notch filters, which calls for an independent setting of the step sizes $\mu_{i}$, $i=1, \ldots, M$, for each individual FxLMS update law. Setting these crucial design parameters is far from trivial in practice and difficult to address with a trial-and-error approach due to the interaction of the various FxLMS adaptation loops. A typical solution to this problem is to employ the normalized version of the FxLMS algorithm, which however increases the computational cost further because of the normalization operations.

The following two methods from the literature address the computational cost issue.

\subsection{Ziegler's system}

In [7] Ziegler proposed a variation of the classical narrowband ANC system that partially compensates the secondary path by accounting for the phase shift it introduces at the controlled frequencies. The adaptive algorithm updates the filter weights so as to minimize the residual error $e^{\prime}(n):$

$$
\begin{aligned}
& w_{0, i}(n+1)=w_{0, i}(n)+\mu_{i} x_{0, i}\left(n-\Delta_{i}\right) e^{\prime}(n), \\
& w_{1, i}(n+1)=w_{1, i}(n)+\mu_{i} x_{1, i}\left(n-\Delta_{i}\right) e^{\prime}(n),
\end{aligned}
$$

for $i=1, \ldots, M$. This update law can be interpreted as a particular case of the FxLMS algorithm where the frequency-dependent secondary-path is approximated as $\hat{S}(z)=z^{-\Delta_{i}}$ (unitary magnitude and phase equal to $\Delta_{i} T_{s}$, where $\Delta_{i}$ is an integer number and $T_{s}$ is the sampling time).

Ziegler's method greatly reduces the computational cost of the basic parallel-form multi-tonal narrowband system, since the reference-filtering operations are reduced to simple integer delays. However, in practice the phase of the secondary path at the $i$ th controlled frequency is only roughly approximated by an integer multiple $\Delta_{i}$ of the sampling period. This results in a spectrally 
inaccurate estimation that, coupled with the lack of magnitude compensation, may significantly degrade the performance of Ziegler's system both in terms of noise cancellation and convergence speed, as shown later on in Section 4.

\subsection{Xiao's system}

In [8] Xiao proposed a variation of the parallel narrowband ANC system that aims at reducing the reference-filtering operations. The reference signals are summed together and only the aggregate signal is convolved with the secondary-path estimate. Finally, the individual filtered reference signals are retrieved using a bank of IIR bandpass filters with the following structure:

$$
H_{b p, i}(z)=\frac{(\rho-1) c_{i} z^{-1}+\left(\rho^{2}-1\right) z^{-2}}{1+\rho c_{i} z^{-1}+\rho^{2} z^{-2}},
$$

for $i=1, \ldots, M$, where $c_{i}=-2 \cos \left(2 \pi f_{i}\right)$ and $\rho$ is a pole-attraction factor. Overall, the resulting system requires less computational effort compared to the basic one since it only performs one convolution with the secondary path model, instead of $M$ convolutions per iteration.

The main issue with this method is that a proper setting of the bandwidth-control parameter $\rho$ becomes critical when the controlled frequencies are close or when their number is large. A large value for $\rho$ determines a sharper bandwidth at the cost of a longer convergence time, while a small value for $\rho$ determines faster convergence but it may ultimately cause system instability since the bandpass filters will not provide adequate separation of close frequencies.

\section{The proposed system}

\subsection{Adaptive notch filtering using local secondary path models}

The order $L^{\prime}$ of the global estimate $\hat{S}(z)$ must typically be quite large to provide an accurate spectral approximation of $S(z)$ over the whole control frequency range. This is also one of the sources of computational complexity of the standard algorithm, which involves many convolution operations with long vectors. On the other hand, convolving a tonal reference signal with $\hat{S}(z)$ actually yields only a phase-shifted and amplified version of the input sinusoid (at least at steadystate). Indeed, to control a given frequency one only needs two parameters to obtain the filtered reference signals, i.e. the magnitude and phase of $S(z)$ at that frequency. This is precisely the rationale behind Ziegler's method of using a pure delay unit to compensate for the phase shift introduced by the secondary path at the controlled frequency, which can be interpreted as an 
ultra-local frequency-wise secondary-path approximation (even though the amplitude is not taken into account and the phase shift compensation is never accurate in practice).

An alternative approach is here explored that exploits the same property without the downside of Ziegler's method. The key idea is to substitute the global $L^{\prime}$-order secondary-path estimate $\hat{S}(z)$ with a set of local $L$-order secondary-path estimates $\hat{S}_{k}(z), k=1, \ldots, K$. These are modeled as short FIR filters $\left(L \ll L^{\prime}\right)$ and provide a local approximation to the secondary path only in a neighborhood of specific frequencies $\tilde{f}_{k}, k=1, \ldots, K$, i.e. $\hat{S}_{k}(f) \approx S(f)$ when $f \in \mathcal{N}\left(\tilde{f}_{k}\right)$ (where $\mathcal{N}\left(\tilde{f}_{k}\right)$ is a suitable neighborhood of $\left.\tilde{f}_{k}\right)$.

More in detail, let $\left[f_{L}, f_{H}\right]$ be a sufficiently large frequency range to include all controlled frequencies. Then, split the interval $\left[f_{L}, f_{H}\right]$ into $K$ frequency subbands $\mathcal{N}\left(\tilde{f}_{k}\right)$ of width $\Delta f=$ $\left(f_{H}-f_{L}\right) / K$ and centered on $\tilde{f}_{k}=f_{L}+(k-1 / 2) \Delta f, k=1, \ldots, K$ :

$$
\mathcal{N}\left(\tilde{f}_{k}\right)=\left[f_{L}+(k-1) \Delta f, f_{L}+k \Delta f\right) .
$$

The parameters $K$ and $L$ must be tuned experimentally depending on the spectral shape of the expected secondary path, so that the phase-estimation error between $\hat{S}_{k}(z)$ and $S(z)$ in $\mathcal{N}\left(\tilde{f}_{k}\right)$ remains at least within the stability bounds. In general, the larger the subbands, the more complex the local models need to be in order to achieve the required accuracy. Notice that the number of local models is totally independent of the number and location of the controlled frequencies, the only required information being the maximum frequency interval spanned by the latter.

In each adaptive notch filter block the filtering of the reference signal is operated employing the appropriate local model $\hat{S}_{k_{i}}(z)$, i.e. the one whose associated subband contains the controlled frequency to which the notch is tuned (see Figure 2). More in detail, each single-tone ANC unit outputs the signal $y_{i}(n)=w_{0, i}(n) x_{0, i}(n)+w_{1, i}(n) x_{1, i}(n)$ and updates its two adaptive weights by convolving its reference signals with the secondary-path model $\hat{S}_{k_{i}}(z)$ associated to the subband $\mathcal{N}\left(\tilde{f}_{k_{i}}\right)$ that includes its reference frequency $f_{i}$, i.e. such that $f_{i} \in \mathcal{N}\left(\tilde{f}_{k_{i}}\right)$. Then, the error signal:

$$
e(n)=d(n)-\sum_{i=1}^{M} y_{i}^{\prime}(n) .
$$

is used to update the ANC units:

$$
\begin{aligned}
& w_{0, i}(n+1)=w_{0, i}(n)+\mu_{i} x_{0, i}^{\prime}(n) e(n), \\
& w_{1, i}(n+1)=w_{1, i}(n)+\mu_{i} x_{1, i}^{\prime}(n) e(n) .
\end{aligned}
$$


where $x_{0, i}^{\prime}(n)$ and $x_{1, i}^{\prime}(n)$ are the reference sinusoidal components, filtered through the appropriate local models $\hat{S}_{k_{i}}(z)$. Notice that, if more than one controlled frequencies fall in the same subband they will be convolved with the same local model. This effectively cuts the down the computational cost associated to the convolution operations required for the filtering of the reference signals, thanks to the reduced length of the local secondary path models.

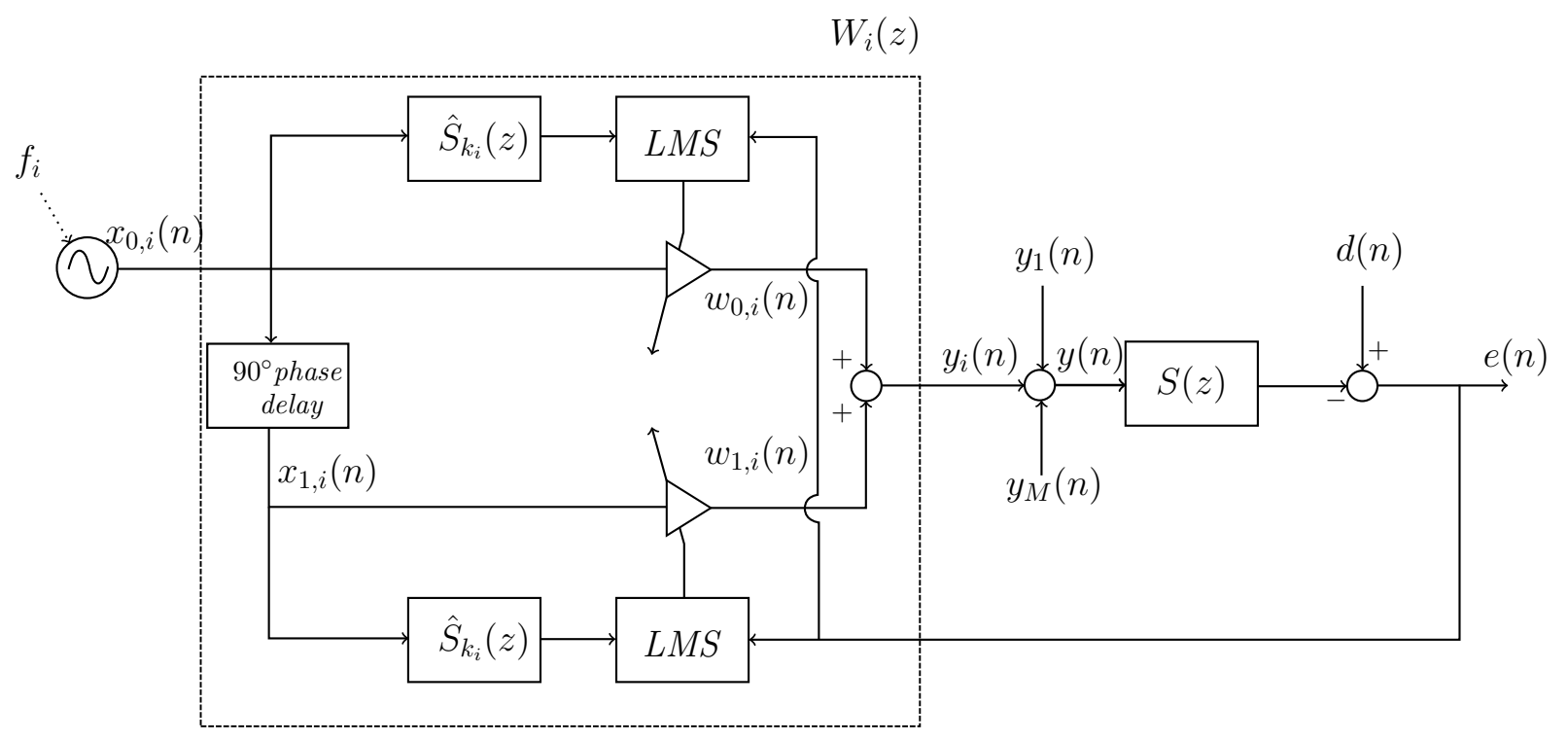

Figure 2: The adaptive notch filters based on local secondary-path models.

\subsection{Modeling of the local secondary paths}

In order to learn the $K$ local secondary paths $\hat{S}_{k}(z), k=1, \ldots, K$, we employ the additional low energy white noise signal exactly as in the traditional method (see Figure 1). To enforce the local model adaptation a frequency pre-filtering approach is applied in the secondary path modeling loops. This method [10] consists in filtering both the input $(a(n))$ and output $(e(n))$ with the same filter and identifying the model using the filtered data. The performance index that is actually minimized is a function of the filtered error, and as a result the accuracy in the filter band is enhanced. Specifically, if a bandpass pre-filter is employed, the resulting model will accurately approximate the secondary path only in the corresponding frequency range. By using a bank of bandpass filters $F_{k}(z), k=1, \ldots, K$, tuned to the subbands of interest, one can then identify all the required local models (see Figure 3). More precisely, the bandpass filters are designed as follows:

$$
F_{k}(z)=\frac{(\rho-1) c_{k} z^{-1}+\left(\rho^{2}-1\right) z^{-2}}{1+\rho c_{k} z^{-1}+\rho^{2} z^{-2}}
$$


for $k=1, \ldots, K$, where $c_{k}=-2 \cos \left(2 \pi \tilde{f}_{k}\right), \tilde{f}_{k}$ being the central frequency of the subband, and $\rho$ is a pole-attraction factor.

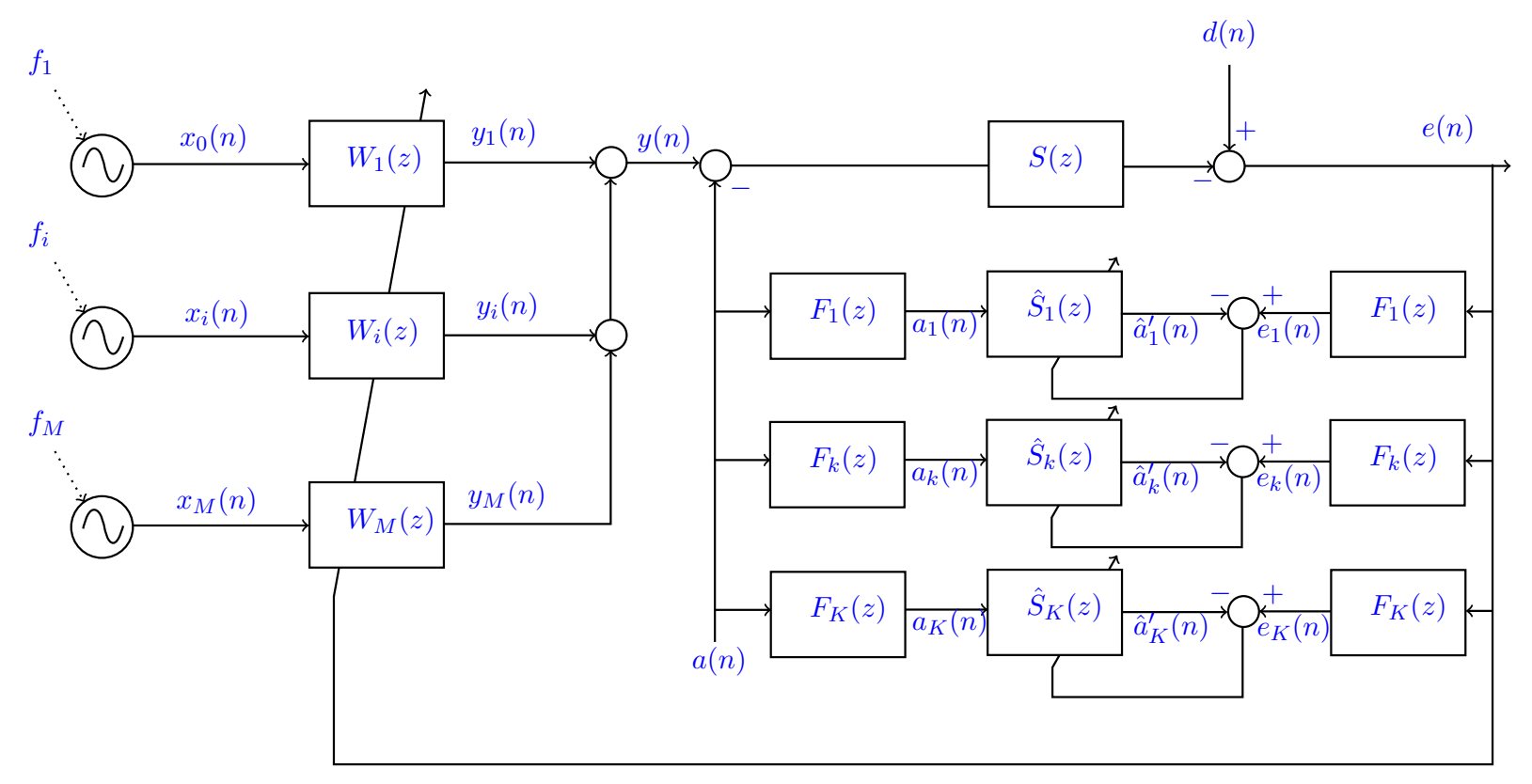

Figure 3: The proposed system including the local secondary path modeling blocks.

Observe that employing a bank of filters in the suggested way does not carry over the same disadvantages of Xiao's system. Indeed, since the central subband frequencies $\tilde{f}_{k}, k=1, \ldots, K$ are independent of the controlled ones and are positioned by design at safe distances, the band-pass filters are well-spaced. On the other hand, in Xiao's system the band-pass filters are selectively designed over the controlled frequencies, which makes them particularly sensitive to their proximity and to the precision with which they are estimated. Such issues are further aggravated in case of time-variability of said controlled frequencies.

In addition, in the proposed scheme the filtering operations associated to the bank of band-pass filters concern only the secondary path modeling and not the main noise cancellation loop, whose convergence characteristics are not affected. Furthermore, consider that the computational cost of these pre-filtering operations can be partially mitigated by limiting the local secondary model adaptation to those models whose associated subbands actually include controlled frequencies.

The LMS update rule for the local secondary path modeling loops becomes:

$$
\hat{\mathbf{s}}_{k}(n+1)=\hat{\mathbf{s}}_{k}(n)+\mu_{s} \mathbf{a}_{k}(n)\left[e_{k}(n)-\hat{a}_{k}^{\prime}(n)\right] .
$$

where $\hat{\mathbf{s}}_{k}(n)=\left[\hat{s}_{k, 0}(n) \ldots \hat{s}_{k, L-1}(n)\right]^{T}$ is the impulse response of the local $L$-order secondary-path 
estimate $\hat{S}_{k}(z), \mathbf{a}_{k}(n)=\left[a_{k}(n) \ldots a_{k}(n-L+1)\right]^{T}$ is the pre-filtered excitation-signal vector, $\mu_{s}$ is the step size, and the two pre-filtered signals $e_{k}(n)$ and $a_{k}(n)$ result from the convolution of $e(n)$ and $a(n)$, respectively, with the bandpass filters $F_{k}(z)$.

The described modeling scheme can be employed both in the offline and online stages. In the offline stage (with the control switched off), one has that

$$
\begin{aligned}
& E_{k}(z)=F_{k}(z)[D(z)+S(z) A(z)], \\
& \hat{A}_{k}(z)=\hat{S}_{k}(z) F_{k}(z) A(z)
\end{aligned}
$$

so that the $z$-transform of the error used in the $k$ th local secondary path modeling loop becomes

$$
E_{k}(z)-\hat{A}_{k}(z)=\left[S(z)-\hat{S}_{k}(z)\right] F_{k}(z) A(z)+F_{k}(z) D(z)
$$

Therefore, since $a(n)$ and $d(n)$ are uncorrelated, the minimum for the $k$ th optimization problem is reached when

$$
\hat{S}_{k}(z) \equiv S(z)
$$

in the frequency range excited by $a_{k}(n), k=1, \ldots, K$, i.e. in the frequency band determined by the filter $F_{k}(z)$.

The same scheme can also be applied in the online phase (when the control is on), in view of the uncorrelation between the excitation and reference signals, which allows the correct convergence of both the local secondary path modeling loops and the ANC loops as in the traditional method. In terms of control performance, a non-colored residual remains due to the injection of the white noise signal $a(n)$.

Besides reducing the computational cost associated to the reference-filtering operations, employing low-order local FIR models also increases the reactivity of the online secondary path modeling subsystem to changes in the secondary path, since larger step sizes may be used (the stability condition demands that the step size be inversely proportional to the length of the filter) and simpler models must be learnt. This aspect is further investigated in Section 4.6.

\subsection{Some remarks on pre-filter design}

Regarding the design of the pre-filters, a trade-off must be solved between the estimation accuracy, which influences the performance of the system, and the model order, which affects both 
the computational cost associated to the reference-filtering operations and the reactivity of the online modeling subsystem to changes in the secondary path. The following factors must be taken into account:

- Choosing a pole-attraction factor $\rho$ close to 1 (i.e. employing a notch-like bandpass filter) could in principle allow one to use local FIR models of order 2 (since the system would only need to model the magnitude and the phase of the secondary path at the excited frequency), but these may be too inaccurate in the rest of the subband (see Figure 4 (top)).

- In the limit, the previous solution turns out to be equivalent to employing pure tonal excitation signals at the central frequencies of each subband. This approach is acceptable in terms of the resulting local secondary path modeling accuracy (provided that the excitation frequencies do not coincide with the controlled ones). However, the control performance is not as satisfactory, since when the controlled frequencies are attenuated, the excitation frequencies -however small- become dominant, resulting in a control system output with a coloured spectrum.

- On the other hand, as $\rho$ gets closer to 0 the bandwidth of the bandpass filter $F_{k}(z)$ becomes larger, thus enlarging the frequency interval on which the model is estimated. In turn, this requires that the order of the model be increased to allow sufficient flexibility to describe the secondary path on the larger frequency interval. The complexity of the associated identification problem is correspondingly increased, which not always results in more accurate results (compare, e.g., the plots in Figure 4).

\subsection{Empirical step size-correction law}

In the standard parallel-form narrowband ANC system, the $i$ th secondary-path convolution can be interpreted as rescaling the step size $\mu_{i}$ by the magnitude of the secondary path frequency response at the corresponding controlled frequency $f_{i}$. Indeed, at steady-state the FxLMS update rule for the first coefficient becomes

$$
\begin{aligned}
w_{0, i}(n+1) & =w_{0, i}(n)+\mu_{i} x_{0, i}^{\prime}(n) e^{\prime}(n)= \\
& =w_{0, i}(n)+\mu_{i} A_{\hat{S}_{k}} \cos \left(2 \pi f_{i} n+\phi_{\hat{S}_{k}}\right) e^{\prime}(n)
\end{aligned}
$$



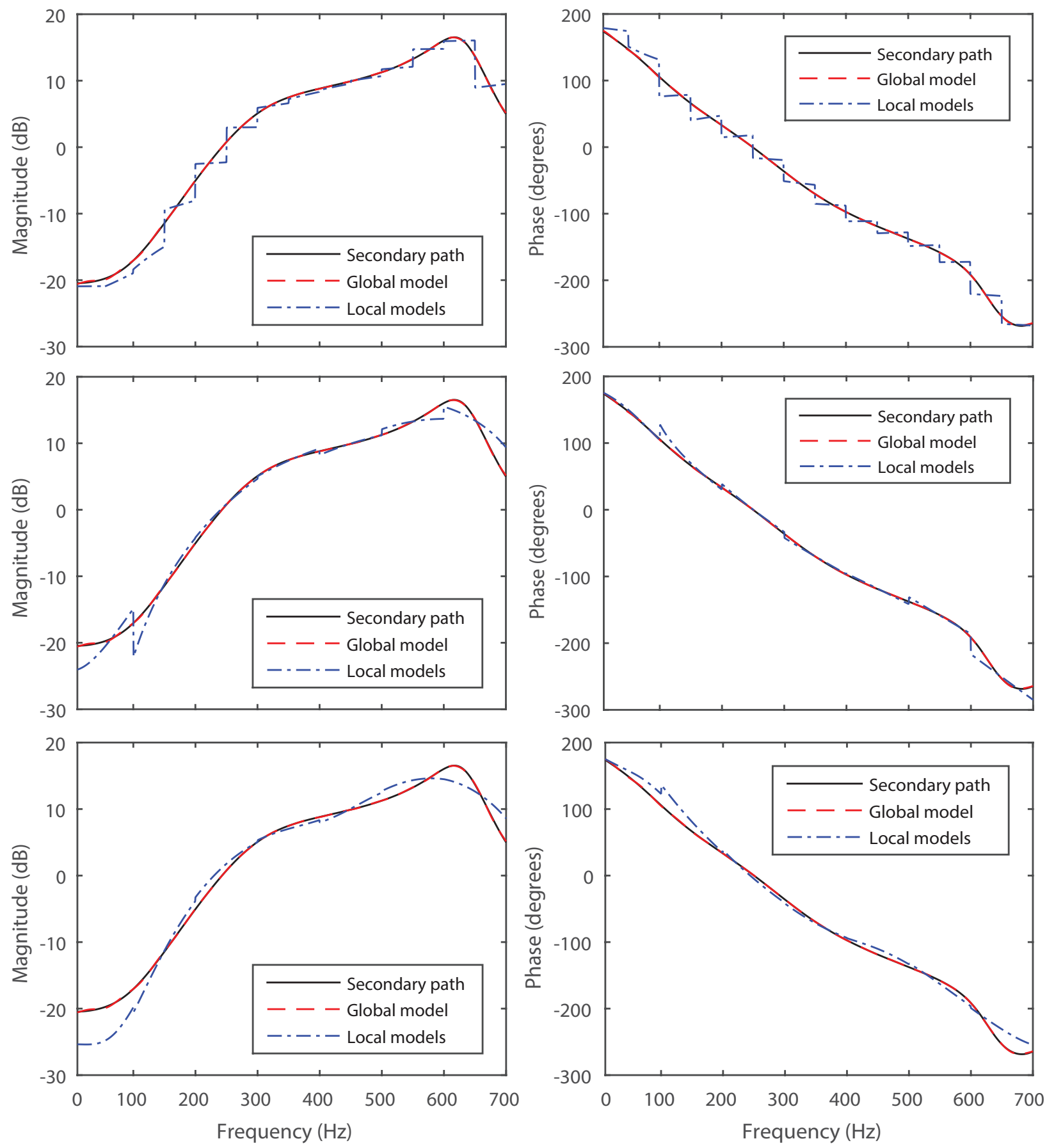

Figure 4: Estimation accuracy of the local secondary path modeling phase, using different pre-filtering and local model size settings: $L=2 \& \rho=0.9995$ (top), $L=25 \& \rho=0.995$ (middle), and $L=40 \& \rho=0.85$ (bottom). For each case, the magnitude (left) and phase (right) of the frequency response are shown for the true secondary path, a global model (of order $\left.L^{\prime}=150\right)$ and the local models (in the respective subbands). 
for $i=1, \ldots, M$, where $A_{\hat{S}_{k}}=\left|\hat{S}_{k}\left(e^{j 2 \pi f_{i}}\right)\right|$ and $\phi_{\hat{S}_{k}}=\angle \hat{S}_{k}\left(e^{j 2 \pi f_{i}}\right)$ and similarly for the second coefficient.

The appropriate tuning of the step size is crucial for the stability of the algorithm and the convergence speed. Unfortunately, it is practically unfeasible to tune each step size manually to compensate for the mentioned scaling effect and enforce comparable convergence speeds on the different ANC modules. For this reason, we here adopt the following empirical step size-correction law:

$$
\mu_{i}=\frac{\alpha}{A_{i}^{\beta}}
$$

for $i=1, \ldots, M$, where $\alpha$ and $\beta$ are two tunable parameters $\left(e . g ., \alpha=\beta=1\right.$ ), and $A_{i}$ can be one of the following alternatives:

- The magnitude of the local secondary-path model at $f_{i}\left(A_{i}=\left|\hat{S}_{k}\left(e^{j 2 \pi f_{i}}\right)\right|\right)$;

- The average magnitude of the local secondary-path model over the frequency bin;

- The maximum magnitude within the frequency bin.

The last two alternatives provide more conservative solutions for what concerns stability. This step size correction law has an analogous effect to the normalized version of the FxLMS algorithm, whose update law amounts to:

$$
w_{j, i}(n+1)=w_{j, i}(n)+\frac{\mu_{i} x_{j, i}^{\prime}(n) e^{\prime}(n)}{x_{0, i}^{\prime}(n)^{2}+x_{1, i}^{\prime}(n)^{2}},
$$

for $i=1, \ldots, M, j=0,1$. Dividing by $x_{0, i}^{\prime}(n)^{2}+x_{1, i}^{\prime}(n)^{2}$ is equivalent to scaling by the reciprocal of the squared magnitude of $\hat{S}_{k}(z)$ at the controlled frequency. However, while the normalization operations have to be carried out at every iteration, the step size correction can be performed only once offline (as long as the controlled frequency or the secondary path do not change).

\section{Simulation examples}

In the following simulations, the primary and secondary paths are 24th order infinite impulse response (IIR) filters taken from [1]. The sampling frequency is $F_{s}=8 \mathrm{kHz}$ and the controlled frequencies are below $700 \mathrm{~Hz}$, so that it is safe to model the secondary path in the frequency range $\left[f_{L}, f_{H}\right]$, with $f_{L}=0 \mathrm{~Hz}$ and $f_{H}=700 \mathrm{~Hz}$. Notice that, since $f_{H}$ is much lower than the Nyquist 
frequency, potentially negative effects on the system noise attenuation capabilities due to sampling are absent.

For ANC purposes, we employ a set of $K=14$ local FIR models of order $L=2$ adapted offline using a white-noise excitation signal with pre-filtering as explained in Section 3.1 and shown in Figure 4 (top). As is apparent from Figure 4 (top), the local secondary paths are not particularly accurate, but this inaccuracy is more than compensated for by the beneficial effects in terms of computational effort and convergence speed that result from the compactness of the involved FIR filters.

The local secondary path modeling blocks are switched off during control operation in the first 5 examples, where a stationary secondary path is assumed and therefore online updating is not necessary. On the other hand, Example 6 tests the reactivity of the modeling blocks online in response to a variation of the secondary path.

\subsection{Example 1: The single-frequency noise case}

We consider first the simplest possible setting, where $d(n)$ contains a single sinusoidal component at frequency $f_{1}=400 \mathrm{~Hz}$. A step size of $\mu_{1}=0.0012$ is used to adapt the coefficients $w_{0,1}(n)$ and $w_{1,1}(n)$. The pole-attraction factor in the bandpass filter of Xiao's system is set to $\rho=0.985$.

The convergence trajectory of the two adaptive coefficients $w_{0,1}(n)$ and $w_{1,1}(n)$ and the residual error $e(n)$ are shown in Figure 5. The basic parallel-form narrowband system, Xiao's system and the proposed system all converge to the optimal solution in about $100 \mathrm{~ms}$, whereas Ziegler's system takes roughly double the time, which shows the possible shortcomings of the discretized delay solution.

The controlled frequency $f_{1}$ lies between two central frequencies $\tilde{f}_{8}=375 \mathrm{~Hz}$ and $\tilde{f}_{9}=425$ $\mathrm{Hz}$ and both $\hat{S}_{8}(z)$ and $\hat{S}_{9}(z)$ display a $10^{\circ}$ phase-estimation error at that frequency (Figure 4 ), which is comparable to the phase error of Ziegler's method $\left(7^{\circ}\right)$. Despite this, the proposed system does not show any noticeable reduction in convergence time with respect to the basic parallel-form narrowband system, because the proposed method accounts for the magnitude of the secondary path as well (which does not affect stability, but influences the convergence speed).

\subsection{Example 2: The multi-tonal noise case}

We next consider the multi-tonal case, where $d(n)$ contains $M=20$ sinusoids with fundamental frequency $f_{1}=30 \mathrm{~Hz}$ and $f_{i}=i f_{1}, i=2, \ldots, 20$. The step sizes are the same in all simulations. 

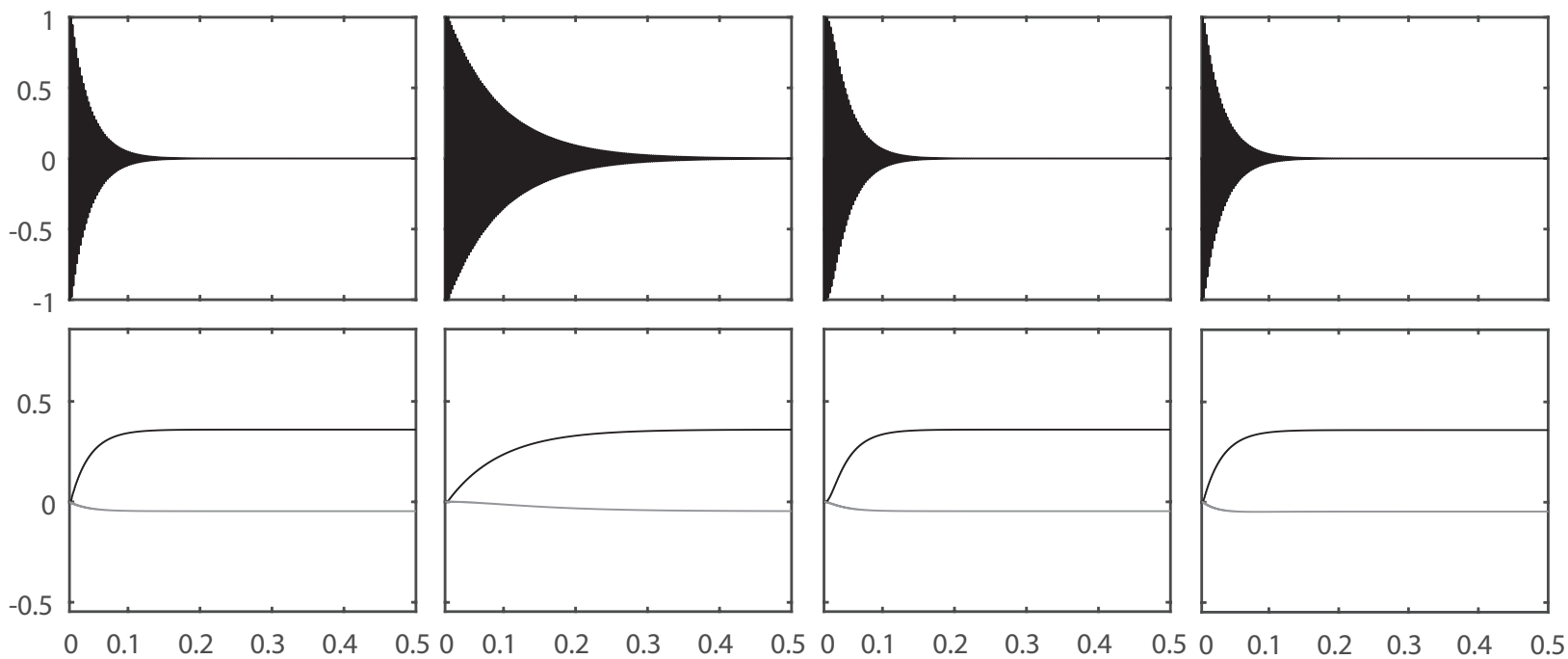

Figure 5: Example 1: Evolution of the residual $e(n)$ (top) and the two weights (bottom). From left to right: Basic system, Ziegler's system, Xiao's system, proposed system.

Figure 6 shows the convergence of the overall error signal and the two adaptive coefficients $w_{15,0}(n)$ and $w_{15,1}(n)$ associated to the fifteenth harmonic $f_{15}$.
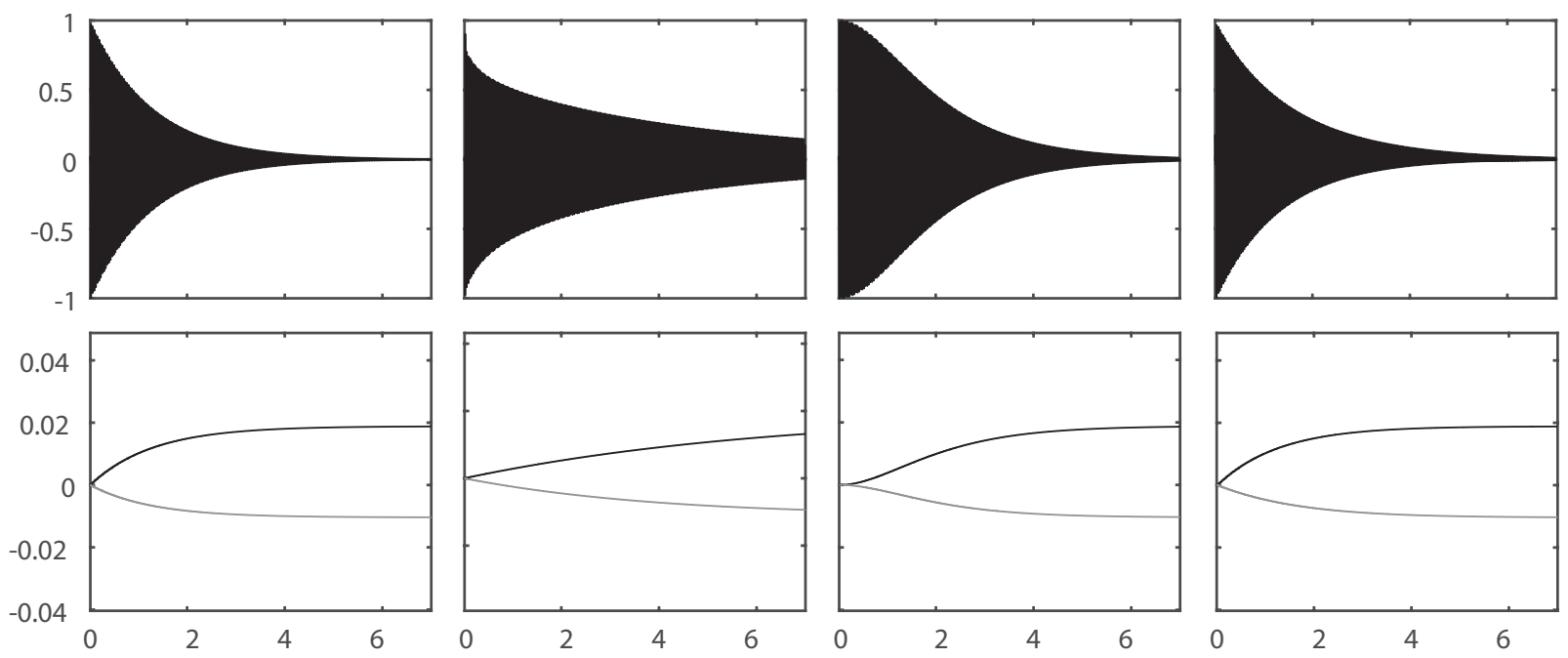

Figure 6: Example 2: Evolution of the residual $e(n)$ (top) and the two weights associated to the fifteenth controlled harmonic (bottom). From left to right: Basic system, Ziegler's system, Xiao's system $(\rho=0.9999)$, proposed system.

It takes the proposed system only slightly longer than the basic parallel-form narrowband system to completely remove all the tonal components. As discussed in the previous sections, both systems suffer from a performance degradation with respect to the single-frequency case due to the fact that each controller uses the same error signal to update its adaptive coefficients. In the 
multiple-frequency case, in fact, the error signal will contain all the tonal components, which will inevitably slow down the convergence of all controllers.

In comparison, the performance of the other two systems is unsatisfactory. For Xiao's system the sensitivity to the bandwidth-control parameter $\rho$ is significant. If $\rho=0.985$, the system is unstable because the bandwidth of the bandpass filters is not sharp enough to separate the filtered reference signals. Conversely, if $\rho=0.9999$, the system is stable, but it takes longer to remove the noise with respect to the proposed and basic systems due to the long time constant associated to the bandpass filters. Ziegler's system is considerably slower than all three systems: convergence is still not achieved after one second.

\subsection{Example 3: Effects of the proximity of controlled frequencies}

In the third example, $d(n)$ contains two close frequencies $f_{1}=400 \mathrm{~Hz}$ and $f_{2}=401 \mathrm{~Hz}$ and the step sizes are $\mu_{1}=\mu_{2}=0.0012$. Figure 7 shows the evolution of the residual error $e(n)$ and the adaptive coefficients $w_{0,1}(n)$ and $w_{1,1}(n)$ associated to $f_{1}$. The proposed and the basic systems
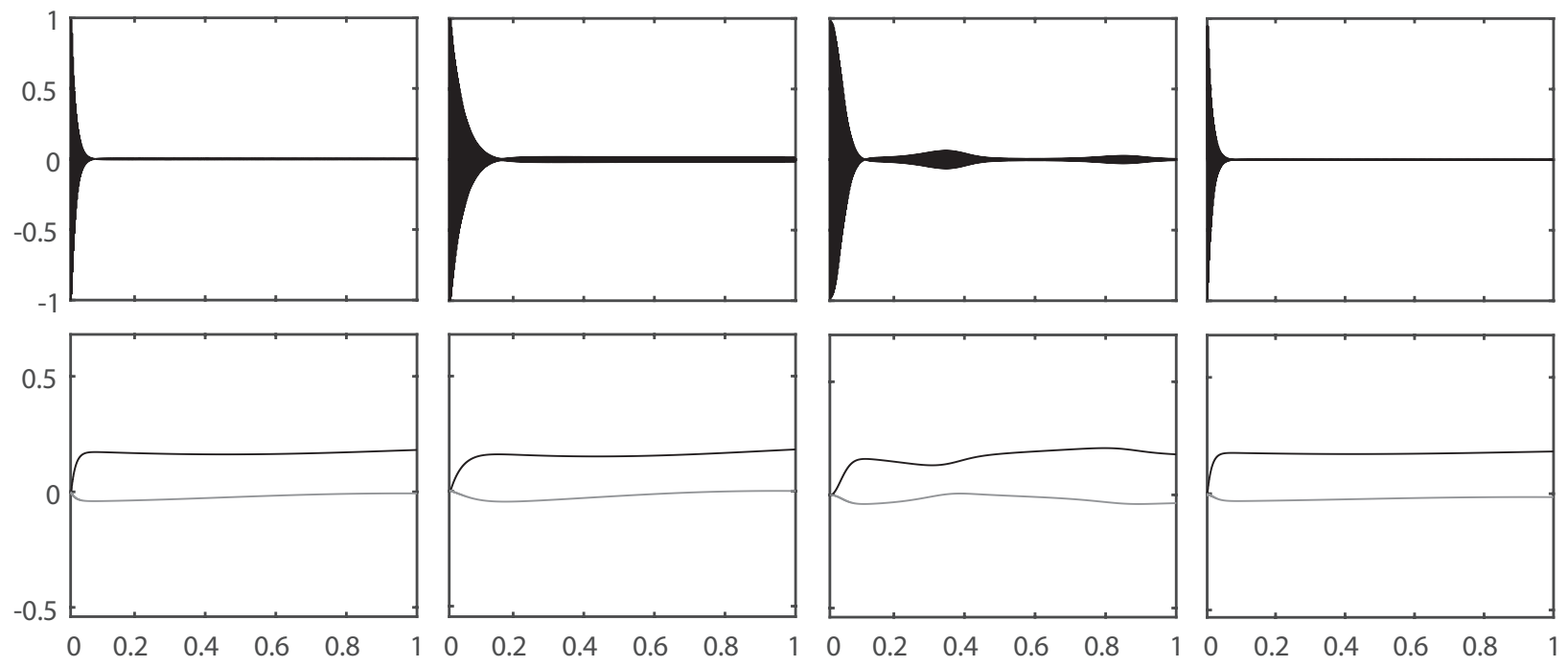

Figure 7: Example 3: Evolution of the residual $e(n)$ (top) and the two weights associated to the fundamental frequency (bottom). From left to right: Basic system, Ziegler's system, Xiao's system $(\rho=0.9999)$, proposed system.

display similar performances, quickly removing the noise except for a small low-level residual. Both methods employ non-filtered tonal components tuned at the controlled frequencies, so that the control performance is not affected by the frequencies' proximity. Xiao's system exhibits some instability-related behaviors which affect the convergence, this time both for low and high values 
of the bandwidth-control parameter $\rho$ (the presented simulation refers to $\rho=0.999$ ). Apparently, the bandpass filtering operations performed in Xiao's scheme cannot neatly separate the two tonal components, which ultimately results in the experienced oscillating behavior. Finally, Ziegler's system exhibits an oscillating behavior as well, although less evident. The noise is rapidly reduced except for an oscillating residual which is damped after about $5 \mathrm{~s}$ (i.e., with the time constant associated to the difference between the two frequencies).

\subsection{Example 4: Frequency mismatch}

A frequency mismatch (FM) between the disturbance frequency content and the frequencies of the controlled tonal components is a known source of deterioration of the performance of an ANC system. Several methods have been proposed in the literature to tackle this issue (see, e.g., [11]). Here, we are merely interested in analyzing the sensitivity of the proposed method to FMs. For this purpose, consider the case where $d(n)$ contains a single frequency $f_{1}=300 \mathrm{~Hz}$ but the reference frequency is set to $f_{1}^{r e f}=303 \mathrm{~Hz}$, which results in a $1 \%$ frequency mismatch. The step size is set to $\mu_{0}=0.0022$. Figure 8 shows the spectral power of the residual noise $e(n)$. The corresponding control performance is illustrated in Figure 8.
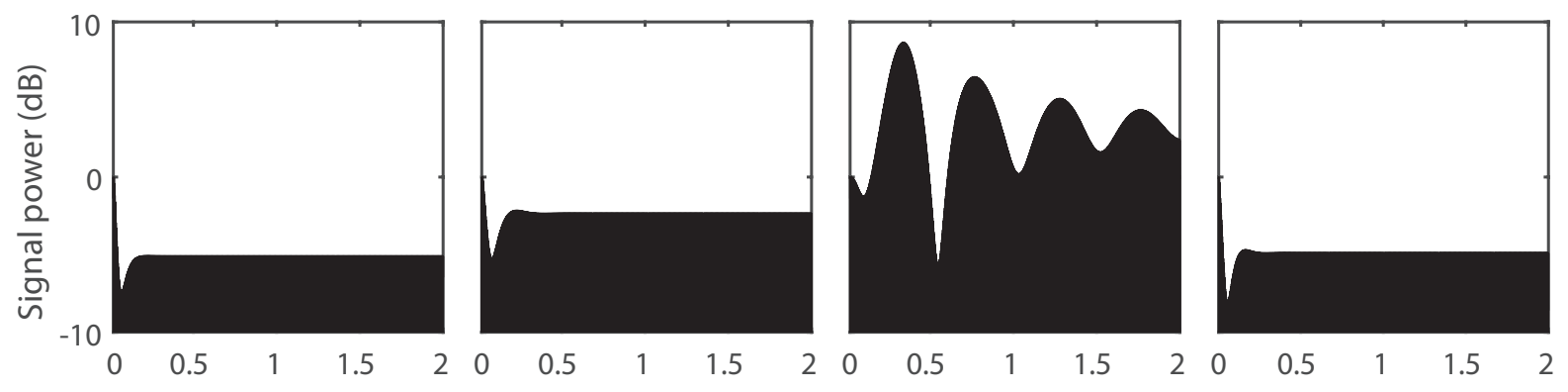

Figure 8: Example 4: Evolution of the power of the residual signal $e(n)$ in the presence of $1 \%$ frequency mismatch. From left to right: Basic system, Ziegler's system, Xiao's system $(\rho=0.9995)$, proposed system.

The proposed system attenuates the noise only by $5 \mathrm{~dB}$, which is the same noise attenuation allowed by the basic parallel-form narrowband system. Indeed, the two schemes are essentially equivalent regarding to how the control signal is generated. The relatively small noise attenuation is in accordance with the theory, since narrowband ANC systems only exhibit a perfect notch at the controlled frequency. Xiao's system exhibits an oscillating behavior that amplifies the noise by 9 $\mathrm{dB}$ during its first oscillation and finally settles to a $3 \mathrm{~dB}$ amplification when $\rho$ is large $(\rho=0.9995$ was used in the simulation), whereas it attenuates the noise by $5 \mathrm{~dB}$ when $\rho$ is small. Indeed, 

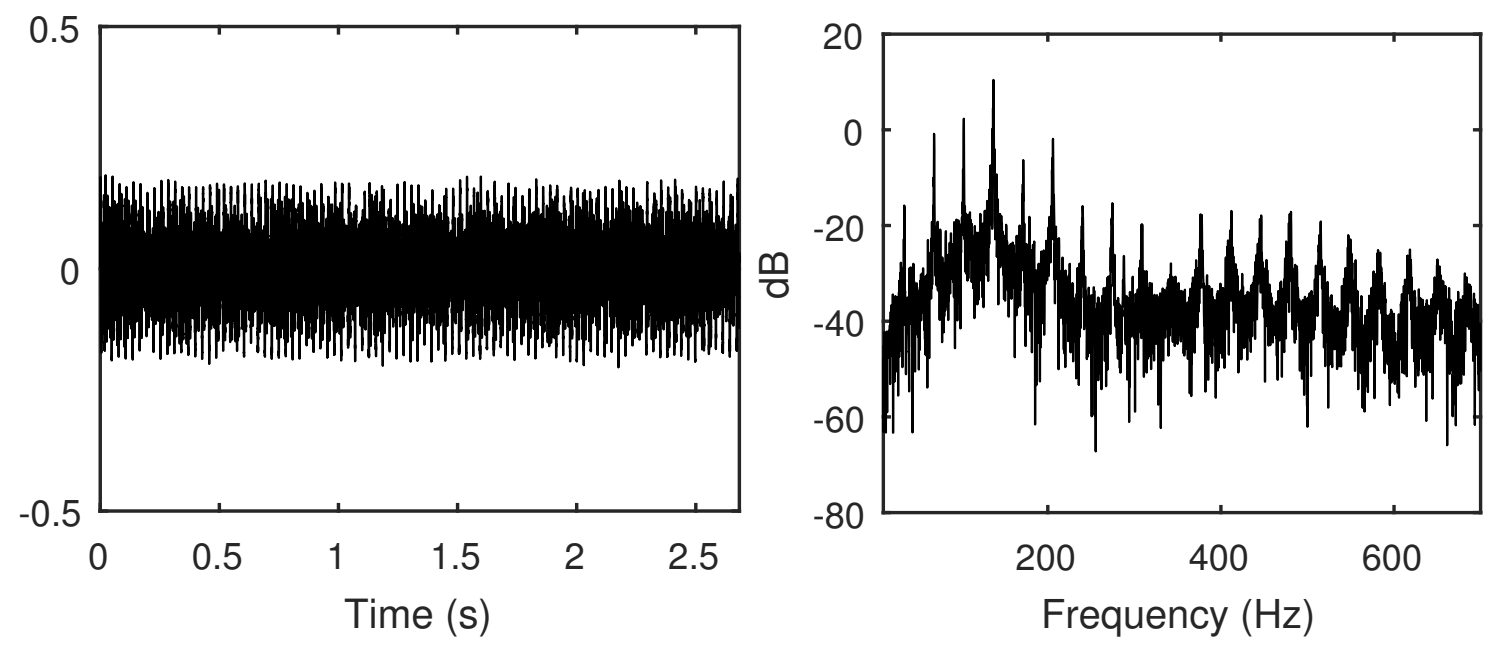

Figure 9: Motorbike noise: Time evolution (left) and power spectrum (right).

with a large $\rho$ the bandpass filtering action of Xiao's system is totally concentrated on the wrong frequency, thereby jeopardizing the control performance. Finally, Ziegler's system attenuates the noise only by $2.5 \mathrm{~dB}$, due to its inaccurate secondary path compensation.

This example merely shows that the proposed method is relatively tolerant of small FM problems, like the traditional method, while the other two methods may be more sensitive for certain choices of their parameters. As already mentioned, several methods can be found in the literature to specifically address the FM issue, but they are beyond the scope of this work.

\subsection{Example 5: A real noise signal}

In the fifth example, the systems are tested against a recording of a motorbike noise, which contains a mixture of low-level broadband noise and several flickering harmonics with fundamental frequency $f_{1}=34.26 \mathrm{~Hz}$. The fundamental frequency was estimated with the autocorrelation method. Figure 9 shows the evolution over time of the motorbike noise signal and its spectrum.

The step sizes are chosen for each controlled frequency according to the empirical step sizecorrection law (19). We set $\alpha=0.003$ and $\beta=2$ for all systems, except Ziegler's one, which required $\alpha=0.0005$ and $\beta=2$ to achieve stability. Table 1 reports the attenuation level (in $\mathrm{dB}$ ) achieved by the four systems for each of the controlled harmonics (the highlighted values indicate the best method for each frequency, negative values denote amplification). Apparently, the basic parallel-form narrowband system displays a strong attenuation at low frequencies, but progressively degrades the performance as the controlled frequency increases, to the point that 

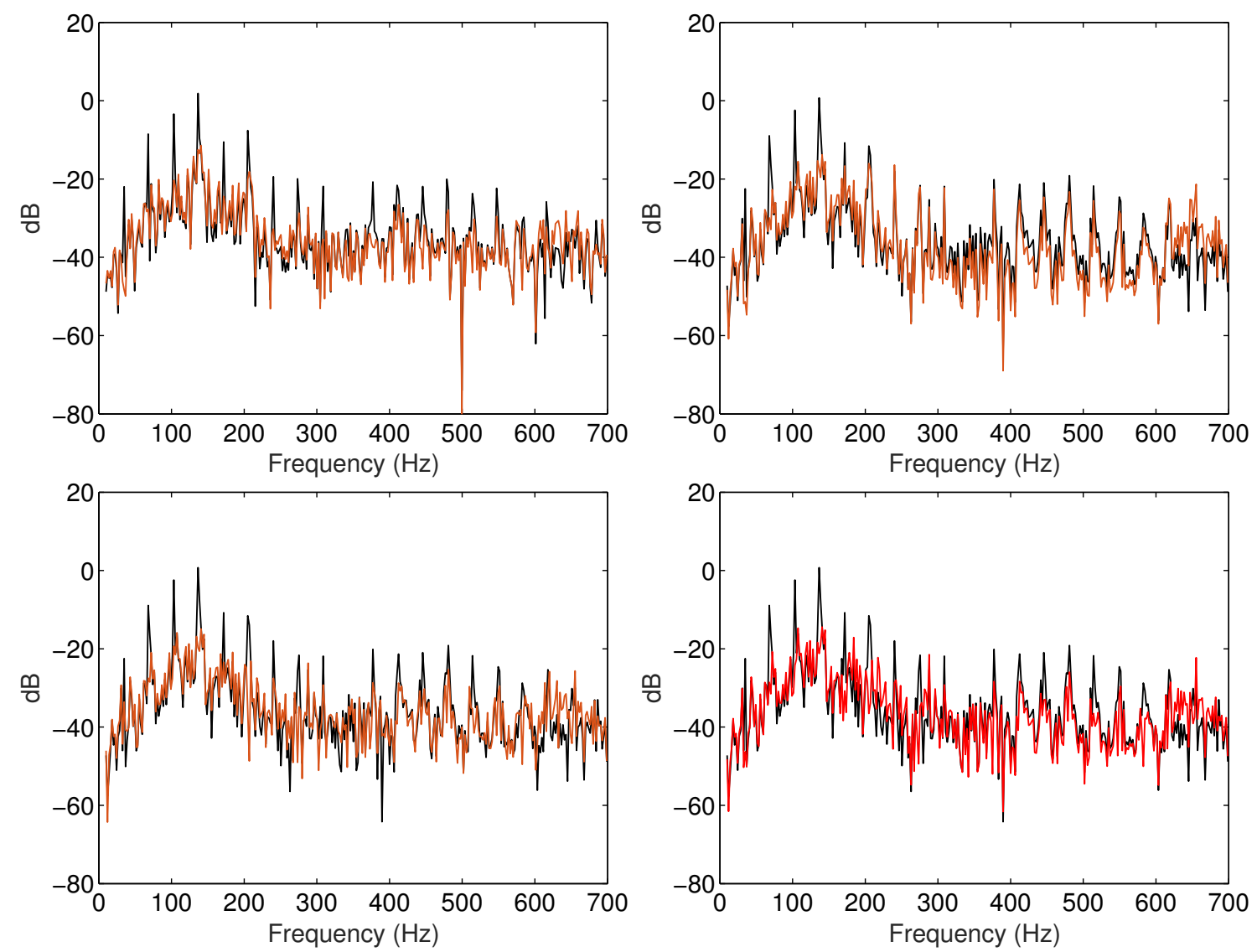

Figure 10: Example 4: Power spectrum of the motorbike noise before noise cancellation (black) and after noise cancellation (red): Basic system (top left), Ziegler's system (top right), Xiao's system ( $\rho=0.9995$, bottom left), proposed system (bottom right).

it completely fails to reduce the highest frequency component. Ziegler's system is effective only for the first few harmonics but then fails to provide adequate attenuation performance. This is probably due to the lower step size needed to ensure its stability. Xiao's system and the proposed one achieve a more balanced reduction over the complete controlled frequency range, the latter being slightly better both in the lower and higher subranges. It is remarkable that the proposed system performs better than all the competitors in the high frequency range. Regarding Xiao's system, the reported results refer to a setting of the bandwidth-control parameter to $\rho=0.9995$ (the system is unstable for $\rho=0.985$ ).

\subsection{Example 6: Tracking a time-varying secondary path}

In the following, we test the online-modeling subsystems in the case of a time-varying secondary path both with a unique global model using the traditional method and with the local models using 


\begin{tabular}{l|cccccccccccccccccccccccccccc}
\hline System & 1 & 2 & 3 & 4 & 5 & 6 & 7 & 8 & 9 & 10 & 11 & 12 & 13 & 14 & 15 & 16 & 7 & 18 & 19 & 20 \\
\hline Traditional & 36.29 & 27.22 & 19.94 & 20.75 & 20.12 & 17.76 & 18.23 & 16.4 & 16.98 & 12.29 & 17.98 & 19.43 & 19.24 & 20.21 & 22.31 & 14.42 & 13.62 & 17.42 & 15.99 & -4.54 \\
Ziegler & 26.46 & 20.86 & 20.65 & 13.32 & 6.68 & 1.45 & -1.33 & -2.33 & -0.29 & 0.41 & 2.23 & 2.47 & 3.05 & 2.98 & 3.37 & 2.7 & 3.22 & -1.55 & 6.6 & 1.68 & \\
Xiao & 12.46 & 19.09 & 17.84 & 15.68 & 13.61 & 12.48 & 9.69 & 13.09 & 10.3 & 11.4 & 16.37 & 17.9 & 31.81 & 21.93 & 21.71 & 24.73 & 15.02 & 20.06 & 17.26 & 17.54 \\
Proposed & 21.68 & 19.35 & 22.56 & 17.99 & 20.94 & 17.29 & 10.59 & 16.08 & 10.24 & 11.71 & 16.23 & 17.37 & 31.42 & 23.98 & 26.17 & 29.44 & 28.57 & 24.6 & 17.27 & 18.33 \\
\hline
\end{tabular}

Table 1: Example 5: Attenuation (in dB) achieved by the four systems for the twenty controlled harmonics.
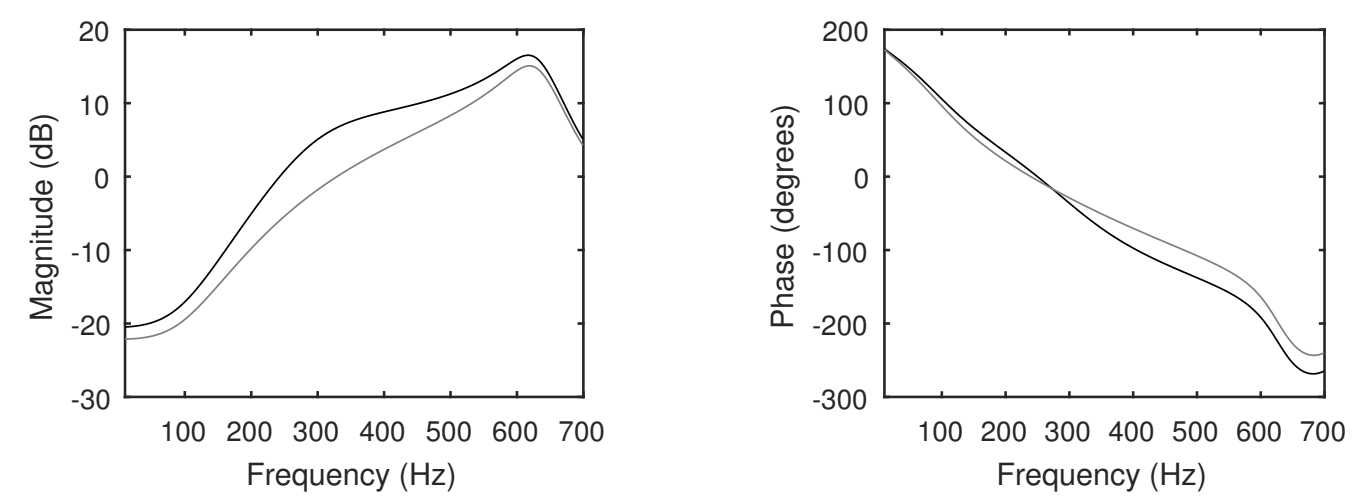

Figure 11: Frequency response of the secondary path before (black line) and after (grey line) the transition.

the proposed method.

The secondary path undergoes a smooth transition between the two frequency response functions represented in Figure 11. This modification is achieved by rotating two complex-conjugate poles of $S(z)$ by $2.25^{\circ}$ (which corresponds to a change in the resonant frequency of $50 \mathrm{~Hz}$ ) and scaling them by a 0.88 factor. Denoting the transfer function of the secondary path as

$$
S(z)=\frac{B(z)}{A(z)}
$$

where $B(z)=b_{0}+b_{1} z^{-1}+\cdots+b_{P} z^{-P}$ and $A(z)=1+a_{1} z^{-1}+\cdots+a_{Q} z^{-Q}$ are suitable polynomials in the unit delay operator $z^{-1}$, the transition is realized by applying the following law:

$$
a_{j}(n+1)=\alpha_{a} a_{j}(n)+\left(1-\alpha_{a}\right) a_{j}^{n e w}
$$

where $A(z)^{\text {new }}$ is the target denominator of the transfer function of the secondary path resulting from the modification. Parameter $\alpha_{a}$ controls the speed of the variation. A value of $\alpha_{a}=0.985$ is used in the simulations, which results in a completion of the transition in about $50 \mathrm{~ms}$. This transition is fast enough to challenge the two alternative online-modeling approaches, but not so abrupt to cause instability.

In the case of the global secondary path estimation, the parameters for the online modeling scheme are set as follows. The variance of the white noise excitation signal is $\sigma_{a}^{2}=0.01$ and the 

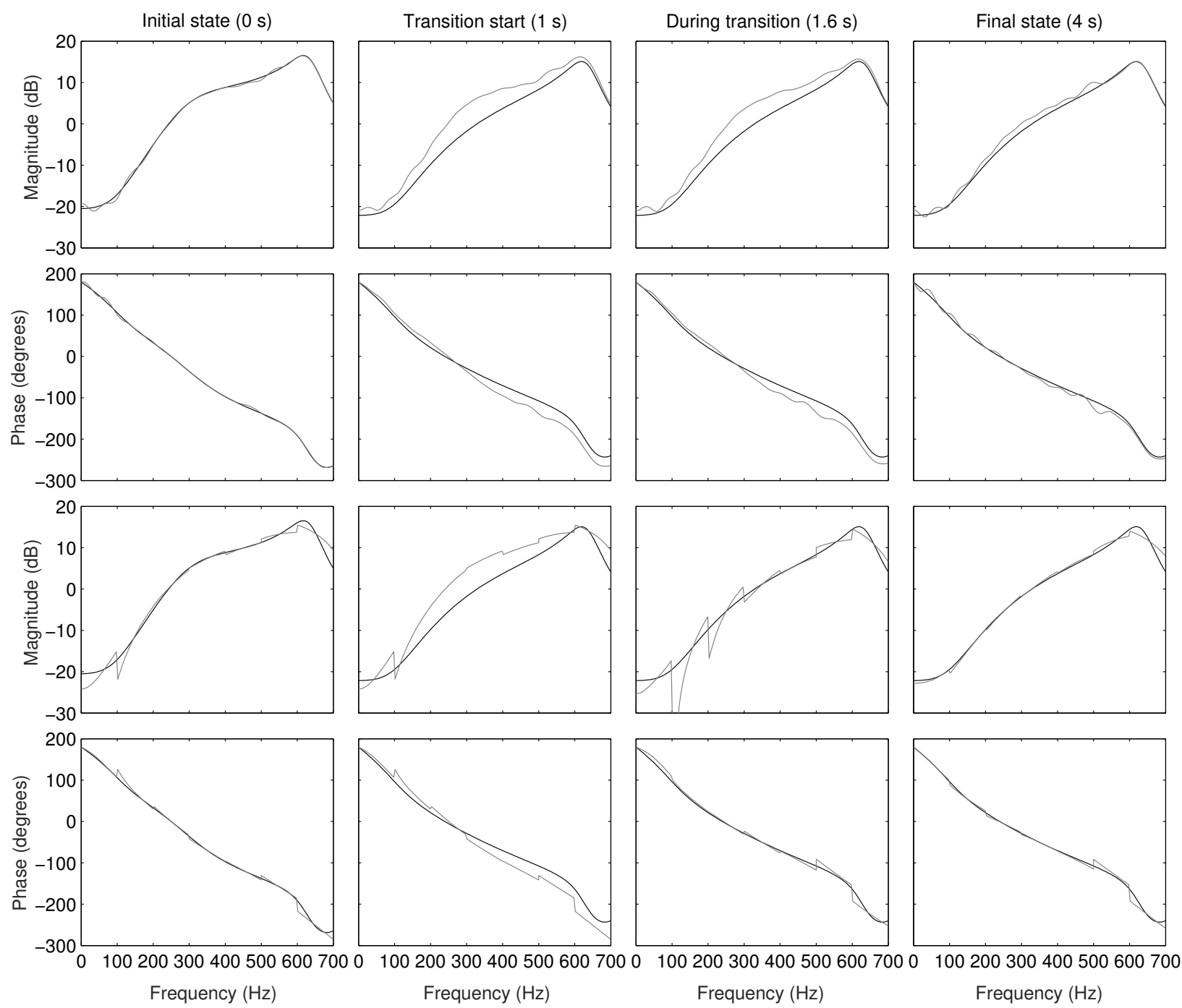

Figure 12: Example 6: Frequency response functions of the secondary path (black) and its global (top two rows) and local (bottom two rows) estimates (grey) at corresponding times (time increases from left to right).

normalized step size is set to $\mu_{s}=0.01$. The identification is carried out with the normalized LMS algorithm. The order of the global estimate $\hat{S}(z)$ is $L^{\prime}=150$. The system is initialized with an accurate global model at time 0. Only one ANC controller is in operation, to remove a single frequency at $f_{1}=500 \mathrm{~Hz}$. Figure 12 (top two rows) shows the tracking capabilities of the online modeling subsystem when the transition takes place at time $t=1 \mathrm{~s}$. After the change, the system takes about $4 \mathrm{~s}$ to adjust the global model $\hat{S}(z)$ to the new secondary path $S(z)$.

For the local estimation scheme, the parameters are set as follows. The normalized step size is set to $\mu_{s}=0.01$ and the variance of the excitation signal is $\sigma_{a}^{2}=0.01$ so that the power of 
the overall excitation signal that is fed to the loudspeaker is the same as in the previous case. As before, the normalized LMS algorithm is employed in the estimation process. The order of the local estimates is $L=25$ and the width of the frequency bins is $\Delta f=100 \mathrm{~Hz}$. Only one ANC controller is active, targeting the removal of the $f_{1}=500 \mathrm{~Hz}$ frequency component. Figure 12 (bottom two rows) shows that most of the local secondary paths converge to the new optimal solution in $0.6 \mathrm{~s}$.

\section{Computational analysis}

Table 2 reports the number of multiplications performed by the considered methods for different numbers of controlled frequencies (considering only the control loop). Ziegler's system is obviously

\begin{tabular}{ccccc}
\hline & Basic & Ziegler & Xiao & Proposed \\
\hline$M$ & $L^{\prime} * M$ & 0 & $L^{\prime}+4 M$ & $L * M$ \\
$M=1$ & 150 & 0 & 154 & 2 \\
$M=2$ & 300 & 0 & 158 & 4 \\
$M=20$ & 3000 & 0 & 230 & 40 \\
\hline
\end{tabular}

Table 2: Number of multiplications per iteration performed by the four systems for varying numbers of controlled frequencies (considering only the control loop).

the most efficient of the four in the online operation, since thanks to the delay blocks the required filtered reference signals are obtained automatically, without a single multiplication. The system however has a significant offline overhead because it must compute the phase lags $\Delta_{i}$ for all possible controlled frequencies, which requires the computation of the discrete Fourier transform of $\hat{S}(z)$ and of its phase delay. Such overhead may affect the online operation as well, should the controlled frequencies vary with time. Xiao's system performs only $L^{\prime}$ multiplications per iteration regardless of the number of controlled frequencies and $4 M$ multiplications due to the $2^{\text {nd }}$-order bandpass IIR filters.

The proposed system reduces the complexity by a factor of $L^{\prime} / L$ with respect to the basic system. In our simulations, we obtain comparable noise attenuation and speed of convergence of the two methods using respectively $L^{\prime}=150$ and $L=2$, which implies that the proposed system reduces the number of performed multiplications by a factor of $L^{\prime} / L=150 / 2=75$. Compared to Xiao's system, the simulations show that the proposed system can both reduce the number of 
multiplications and perform better.

Regarding the cost of the online-modeling subsystem, the proposed system has an additional cost due to the pre-filtering operations. This additional cost amounts to $8 K$ multiplications per iteration (although as explained in Section 3.1 only the local models employed by an ANC unit actually need to be adapted online).

\section{Conclusions}

In this paper, a novel narrowband ANC system has been proposed for multi-tonal noise signals. The system is based on the usage of low-order local secondary path models estimated on adjacent subbands instead of the usual large global model. This turns out to be beneficial in terms of the computational cost, without affecting the noise attenuation performance. Furthermore, the low order of the local secondary paths improves the reactivity to changes in the secondary path, which makes the resulting system more robust to time-varying environments, compared to systems that rely on a global high-order estimate of the secondary path. An empirical step size-correction law is also proposed to automatically tune the step size of each controller in parallel-form narrowband ANC systems.

Simulations show that the proposed system can reduce the computational cost of the referencefiltering operations by more than one order of magnitude compared to the basic parallel-form narrowband ANC system, while preserving the same performance. It also outperforms two other well-known cost-effective systems in specific challenging situations.

\section{References}

[1] S. M. Kuo and D. R. Morgan, Active Noise Control Systems: Algorithms and DSP Implementations. WileyInterscience, 1996.

[2] S. Elliott, Signal Processing for Active Control. Academic Press, 2001.

[3] B. Widrow, J. R. Glover, J. M. McCool, J. Kaunitz, C. S. Williams, R. H. Hearn, J. R. Zeidler, J. E. Dong, and R. C. Goodlin, "Adaptive noise cancelling: Principles and applications," Proceedings of the IEEE, vol. 63, pp. 1692-1716, Dec 1975.

[4] J. Burgess, "Active adaptive sound control in a duct: A computer simulation," Journal of the Acoustical Society of America, vol. 70, no. 3, pp. 715-726, 1981.

[5] C.-Y. Chang and S. Kuo, "Complete parallel narrowband active noise control systems," IEEE Transactions on Audio, Speech, and Language Processing, vol. 21, pp. 1979-1986, Sept 2013. 
[6] J. Cheer and S. Elliott, "Comments on complete parallel narrowband active noise control systems," IEEE/ACM Transactions on Audio, Speech, and Language Processing, vol. 22, pp. 995-996, May 2014.

[7] E. J. Ziegler, "Selective active cancellation system for repetitive phenomena," 1989.

[8] Y. Xiao, "New narrowband active noise control systems requiring considerably less multiplications," in $16^{t h}$ European Signal Processing Conference, pp. 1-5, Aug 2008.

[9] J. Glover, "Adaptive noise canceling applied to sinusoidal interferences," IEEE Transactions on Acoustics, Speech, and Signal Processing, vol. 25, pp. 484-491, Dec 1977.

[10] T. Söderström and P. Stoica, System identification. London: Prentice-Hall, 1989.

[11] H. Jeon, T. Chang, and S. Kuo, "Analysis of frequency mismatch in narrowband active noise control," IEEE Transactions on Audio, Speech, and Language Processing, vol. 18, no. 6, pp. 1632-1642, 2010.

[12] R. Delegà, "A novel cost-effective parallel narrowband ANC system with local secondary path estimation," Master's thesis, Politecnico di Milano, 2016.

[13] S. M. Kuo and D. R. Morgan, "Active noise control: a tutorial review," Proceedings of the IEEE, vol. 87, pp. 943-973, Jun 1999.

[14] P. Lueg, "Process of silencing sound oscillations," 1936. 


\section{List of Figures}

1 Basic narrowband ANC system with online secondary path modeling. . . . . . . 5

2 The adaptive notch filters based on local secondary-path models. . . . . . . . . . . 10

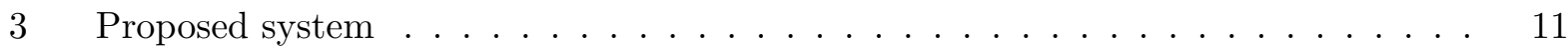

4 Estimation accuracy of the local secondary path modeling phase, using different pre-filtering and local model size settings: $L=2 \& \rho=0.9995$ (top), $L=25 \&$ $\rho=0.995$ (middle), and $L=40 \& \rho=0.85$ (bottom). For each case, the magnitude (left) and phase (right) of the frequency response are shown for the true secondary path, a global model (of order $L^{\prime}=150$ ) and the local models (in the respective

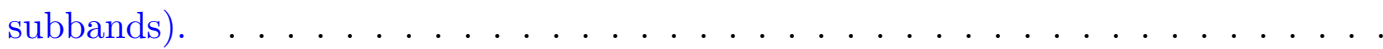

$5 \quad$ Example 1: Evolution of the residual $e(n)$ (top) and the two weights (bottom). From left to right: Basic system, Ziegler's system, Xiao's system, proposed system. . . .

6 Example 2: Evolution of the residual $e(n)$ (top) and the two weights associated to the fifteenth controlled harmonic (bottom). From left to right: Basic system, Ziegler's system, Xiao's system $(\rho=0.9999)$, proposed system. . . . . . . . . .

$7 \quad$ Example 3: Evolution of the residual $e(n)$ (top) and the two weights associated to the fundamental frequency (bottom). From left to right: Basic system, Ziegler's system, Xiao's system $(\rho=0.9999)$, proposed system. $\ldots \ldots \ldots \ldots$

8 Example 4: Evolution of the power of the residual signal $e(n)$ in the presence of 1\% frequency mismatch. From left to right: Basic system, Ziegler's system, Xiao's

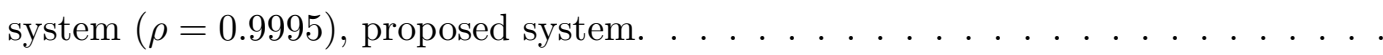

9 Motorbike noise: Time evolution (left) and power spectrum (right) . . . . . . .

10 Example 4: Power spectrum of the motorbike noise before noise cancellation (black) and after noise cancellation (red): Basic system (top left), Ziegler's system (top right), Xiao's system ( $\rho=0.9995$, bottom left), proposed system (bottom right). .

11 Frequency response of the secondary path before (black line) and after (grey line) the transition. . . . . . . . . . . . . . . . . . . . .

12 Example 6: Frequency response functions of the secondary path (black) and its global (top two rows) and local (bottom two rows) estimates (grey) at corresponding times $($ time increases from left to right $) \ldots \ldots \ldots \ldots$ 